\title{
What chance a malaria vaccine?
}

\author{
As hopes recede that traditional means of controlling malaria will succeed, the promise and \\ problems, both scientific and political, of malaria vaccines are coming into focus.
}

VACCINES against malaria are suddenly all the fashion. Research groups, the World Health Organization (WHO) and biotechnology companies seem eager to exploit recombinant DNA and monoclonal antibody techniques to isolate and produce those parasite antigens that might form the basis of vaccines. So it is important to realize that the current interest in vaccines has also been stimulated by the declining effectiveness of earlier methods of control.

The optimism of past decades that a combination of mosquito eradication and treatment of the disease would provide effective control of malaria has now been largely dispelled, several success stories notwithstanding. Partly because of indiscriminate use, these methods are no longer so effective. The problem is resistance - to the insecticides, mainly DDT, used to erradicate mosquitoes (successfully in Italy, Greece and parts of South America) and to chloroquine, once the best of the few drugs active against the disease but fast becoming ineffective as resistance to it has has spread from Asia to South America and now to Africa. (Conceivably others will in time be developed on the basis of the newly discovered susceptibility of the malarial parasite to oxygen or peroxide radicals see Nature 3 March, p.19.) With only one new drug, mefloquine, on the horizon the immediate prospects of controlling malaria look bleak and it is spreading back to some areas at one time freed of the disease.

The new enthusiasm for vaccines is thus understandable, particularly with the prospect that biotechnology will facilitate their production. The first success of the biotechnological approach is reported on page 536 of this issue.

The success is a just reward for Dr Ruth Nussenzweig's persistence in her studies of the sporozoite form of the malaria parasite, which is injected into the bloodstream by a mosquito bite. By all accounts, it puts the prospect of a sporozoite vaccine about two years ahead of a vaccine against forms of the parasite arising at later stages in its life-cycle. But, not least because the cloned gene is from a species of Plasmodium that causes malaria in monkeys rather than one of the four that infects man, the work is more of a hop than a jump towards an effective vaccine.

In any case, there are doubts that a sporozoite vaccine alone will ever be effective in itself. The problem is that the 1,000 or so sporozoites injected by a mosquito bite are, like ballistic missiles, difficult targets and apt to cause great damage even if only one gets through. There is only about $\mathbf{3 0}$ minutes from their entry into the bloodstream in which to destroy the sporozoites before they go to ground in the liver. Once there, a process of development and replication leads to the production of many thousands of merozoites which are released into the bloodstream to enter red cells, continue multiplication and cause the symptoms of malaria. To a first approximation, the final number of merozoites is the same however many sporozoites enter the liver, so unless a vaccine against sporozoites removed them all, the course of the disease will be slower but the end result the same.

Even so, the sporozoite vaccine may have one practical advantage over the merozoite vaccine which many favour - it may work without the need for an adjuvant, a substance that enhances the antibody response to an antigen with which it is given. In several studies, including one on people, protection against malaria has been induced simply by prior exposure to sporozoites rendered incapable of further development by irradiation. Whether the purified surface antigen of the sporozoite will also work in this way is not yet known.

Merozoites, by contrast, require an adjuvant and none that is available is acceptable for use in people. A different problem confronts those developing the third possible kind of vaccine - that against the gametocyte stage of the parasite which develops from the merozoite and which continues the life cycle when taken back into mosquitoes. For such a vaccine, however effective, would do nothing for those vaccinated since it is aimed only at preventing transmission of the disease.

Over and above these problems hovers a general cloud of doubt about the likely effectiveness of any vaccine against malaria, given the persistence of the disease in the face of circulating antibodies in many patients.

Even so, and despite the practical difficulties in developing countries of vaccinating children in their first year of life when most malaria infections and fatalities occur - all three vaccines are still worth pursuing. If, however, the sporozoite vaccine is anything to go by, progress will be complicated not only by scientific and technological problems but also by the clash of political and commercial interests.
Malaria is a disease of developing countries influential on WHO which supports the majority of malaria research. Not surprisingly, WHO insists that discoveries stemming from research it sponsors be freely available to its members. That presents difficulties when, as in the case of Dr Nussenzweig's work, the institution in which she is employed takes out patents and then enters into negotiations with a biotechnology company (in this case, Genentech) that demands exclusive rights on any vaccine produced in return for carrying out the further development of the product.

It is not yet clear to what extent a clash between the vested interests of the company and WHO accounted for the delay and eventual breakdown of negotiations in this instance (the company maintains its lost interest for other reasons - see Nature 31 March, p.366).

It is certain that delay cannot be in the interests of the 200 million infected with malaria at any one time or the 2 million who die from it each year. Since biotechnology companies are in business to make profits and since there can, as yet, be no assurance that a malaria vaccine will be profitable, it is understandable that a company should drive a hard bargain. It is equally understandable that politicians from developing countries will resent the prospect of Californian entrepeneurs growing rich on their problems. How is that dilemma to be resolved?

The best option for WHO must be to persuade the institution holding patent rights to do no more initially than to contract with a biotechnology company to produce vaccine for initial trials. WHO would have to foot the bill for that and thereafter, if the vaccine looked promising, would probably be best off striking a commercial deal with a company (not necessarily the same) to produce the vaccine, to market it in some countries but also to license it to any other members of WHO. It might also be made a condition of WHO support that it becomes the beneficiary of any patents that may arise. For if there are objections to the prospect of a risk-taking company making a profit from the development of a WHO-supported discovery, surely there are even more objections to the possibility that the institution where any such discovery is made should, without taking any risk, stand to profit at the expense of WHO. 